\title{
A sample of relatively unstudied star clusters in the Large Magellanic Cloud: fundamental parameters determined from Washington photometry ${ }^{\star}$
}

\author{
T. Palma ${ }^{1,2}$, J. J. Clariá ${ }^{1,2}$, D. Geisler ${ }^{3}$, A. E. Piatti ${ }^{2,4}$, and A. V. Ahumada ${ }^{1,2}$ \\ 1 Observatorio Astronḿico, Universidad Nacional de Córdoba, Laprida 854, Córdoba, CP 5000, Argentina \\ e-mail: tali@mail.oac.uncor.edu \\ 2 Consejo Nacional de Investigaciones Científicas y Técnicas (CONICET) Av. Rivadavia 1917, C1033AAJ, \\ Ciudad Autónoma de Buenos Aires, República Argentina \\ 3 Grupo de Astronomía, Departamento de Astronomía, Universidad de Concepción, Casilla 160-C, Concepción, Chile \\ ${ }^{4}$ Instituto de Astronomía y Física del Espacio, CC67, Suc. 28, 1428 Ciudad de Buenos Aires, Argentina
}

Received 22 November 2012 / Accepted 16 April 2013

\begin{abstract}
Aims. To enlarge our growing sample of well-studied star clusters in the Large Magellanic Cloud (LMC), we present CCD Washington $C T_{1}$ photometry to $T_{1} \sim 23$ in the fields of twenty-three mostly unstudied clusters located in the inner disc and outer regions of the LMC.

Methods. We estimated cluster radii from star counts. Using the cluster Washington $\left(T_{1}, C-T_{1}\right)$ colour-magnitude diagrams, statistically cleaned from field star contamination, we derived cluster ages and metallicities from a comparison with theoretical isochrones of the Padova group. Whenever possible, we also derived ages using $\delta T_{1}$ - the magnitude difference between the red giant clump and the main sequence turn off - and estimated metallicities from the standard giant branch procedure. We enlarged our sample by adding clusters with published ages and metallicities determined on a similar scale by applying the same methods. We examined relationships between their positions in the LMC, ages, and metallicities.

Results. We find that the two methods for age and metallicity determination agree well with each other. Fourteen clusters are found to be intermediate-age clusters $(1-2 \mathrm{Gyr})$, with $[\mathrm{Fe} / \mathrm{H}]$ values ranging from -0.4 to -0.7 . The remaining nine clusters turn out to be younger than $1 \mathrm{Gyr}$, with metallicities between 0.0 and -0.4 .

Conclusions. Our 23 clusters represent an increase of $\sim 30 \%$ in the current total amount number of well-studied LMC clusters using Washington photometry. In agreement with previous studies, we find no evidence for a metallicity gradient. We also find that the younger clusters were formed closer to the LMC centre than the older ones.
\end{abstract}

Key words. techniques: photometric - galaxies: star clusters: general - Magellanic Clouds

\section{Introduction}

The Large Magellanic Cloud (LMC) has become one of the most-studied objects in the past three decades. In particular, studying the LMC star cluster system advances our understanding of the chemical enrichment and star-formation history of this galaxy as a whole (e.g., Baumgardt et al. 2013), with the important caveat that a large portion of the LMC's history cannot be studied via clusters because of the infamous age gap (Geisler et al. 1997). However, the number of well-studied clusters in the LMC still constitutes a very small fraction of those that have been catalogued, and thorough investigations of even a handful of clusters can significantly improve our knowledge of the chemical enrichment history of this critical galaxy.

The current study represents a step forward in the systematic study of LMC clusters carried out as uniformly as possible using the Washington photometric system. Although initially developed for late-type stars and old stellar populations (Canterna 1976), the Washington system has been widely

* Tables of the photometry for each cluster are only available at the CDS via anonymous ftp to cdsarc.u-strasbg.fr (130.79.128.5) or via

http://cdsarc.u-strasbg.fr/viz-bin/qcat?]/A+A/555/A131 applied to intermediate-age and old clusters in the Galaxy and in the Magellanic Clouds (e.g., Geisler et al. 1997; Geisler \& Sarajedini 1999; Piatti et al. 2003b; Piatti 2012). It is our purpose to derive ages and metallicities for a sample of 23 mostly unstudied LMC star clusters with the aim of adding them to our growing sample of well-studied clusters. The reasons why we have chosen to work in this photometric system and its advantages for this type of study have already been described in previous papers (e.g., Geisler et al. 1997; Piatti et al. 2011).

The cluster sample is presented in Sect. 2. The observations and reductions are described in Sect. 3. The procedure followed to estimate cluster radii from the stellar density profiles is described in Sect. 4. We also include in this section the method applied to minimize the field-star contamination in the Washington $\left(T_{1}, C-T_{1}\right)$ colour-magnitude diagrams (CMDs) and the estimation of the cluster fundamental properties. A brief analysis and discussion of the results is presented in Sect. 5, while Sect. 6 summarizes our results.

\section{Cluster sample}

After a careful revision of the Washington wide-field images of 21 LMC regions (see Sect. 3), we selected those star clusters 
Table 1. Observed star clusters in the LMC.

\begin{tabular}{|c|c|c|c|c|c|c|c|}
\hline Star cluster $^{a}$ & $\begin{array}{c}\alpha_{2000} \\
(\mathrm{hms})\end{array}$ & $\begin{array}{l}\delta_{2000} \\
\left({ }^{\prime \prime \prime \prime}\right)\end{array}$ & $\begin{array}{c}l \\
\left(^{\circ}\right)\end{array}$ & $\begin{array}{l}b \\
\left(^{\circ}\right)\end{array}$ & $\begin{array}{l}r^{b} \\
\left({ }^{\prime}\right)\end{array}$ & $\begin{array}{l}r_{\mathrm{cls}} \\
(\mathrm{px})\end{array}$ & $\begin{array}{l}r_{\mathrm{cls}} \\
(\mathrm{pc})\end{array}$ \\
\hline SL 33, LW 59, KMHK 91 & 044625 & -723406 & 284.717 & -34.986 & 0.55 & 200 & 13.1 \\
\hline SL 41, LW 64, KMHK 105 & 044730 & -723518 & 284.704 & -34.903 & 0.72 & 220 & 14.4 \\
\hline KMHK 123 & 044900 & -723824 & 284.713 & -34.780 & 0.30 & 110 & 7.2 \\
\hline КМHK 128 & 044914 & -720324 & 285.177 & -34.613 & 0.26 & 110 & 7.2 \\
\hline LW 69, КMHK 137 & 044939 & -721453 & 284.246 & -34.874 & 0.32 & 120 & 7.8 \\
\hline KMHK 151 & 045021 & -724939 & 284.881 & -34.619 & 0.27 & 170 & 11.1 \\
\hline SL 54, LW 78, KMHK 162 & 045048 & -723436 & 284.582 & -34.677 & 0.55 & 200 & 13.1 \\
\hline SL 73, LW 86, KMHK 214 & 045245 & -723104 & 284.454 & -34.561 & 0.34 & 190 & 12.4 \\
\hline SL 72, LW 87, KMHK 217 & 045254 & -721021 & 284.054 & -34.667 & 0.42 & 160 & 10.5 \\
\hline BSDL 594, LOGLE 87 & 050553 & -670258 & 277.678 & -35.039 & 0.42 & 140 & 9.2 \\
\hline BSDL 654,LOGLE 123 & 050721 & -664945 & 277.377 & -34.949 & 0.22 & 75 & 4.9 \\
\hline BSDL 665, LOGLE 130 & 050747 & -664753 & 277.329 & -34.914 & 0.22 & 60 & 3.9 \\
\hline BSDL 675, LOGLE 134 & 050756 & -672128 & 277.990 & -34.776 & 0.29 & 90 & 5.9 \\
\hline HS 130, KMHK 588 & 050915 & -674159 & 278.362 & -34.577 & 0.27 & 90 & 5.9 \\
\hline BSDL 761 & 051002 & -664157 & 277.155 & -34.717 & 0.32 & 90 & 5.9 \\
\hline BSDL 779, LOGLE 182 & 051032 & -665624 & 277.428 & -34.619 & 0.22 & 80 & 5.2 \\
\hline HS 156, H88-190, KMHK 632, LOGLE 199 & 051111 & -673736 & 278.227 & -34.414 & 0.25 & 120 & 7.8 \\
\hline HS 178, KMHK 667 & 051348 & -663712 & 276.970 & -34.367 & 0.33 & 120 & 7.8 \\
\hline LW 211, KMHK 901 & 052527 & -733413 & 284.858 & -31.979 & 0.33 & 160 & 10.5 \\
\hline C11 & 055048 & -714228 & 282.371 & -30.397 & 0.20 & 150 & 9.8 \\
\hline BSDL 3158 & 055211 & -715130 & 282.533 & -30.276 & 0.46 & 220 & 14.4 \\
\hline KMHK 1702 & 061356 & -723019 & 283.190 & -28.586 & 0.31 & 100 & 6.5 \\
\hline SL 870, LW 440, KMHK 1705 & 061428 & -723634 & 283.310 & -28.546 & 0.58 & 230 & 15.0 \\
\hline
\end{tabular}

Notes. ${ }^{(a)}$ Cluster identifications from (SL): Shapley \& Lindsay (1963); (LW): Lynga \& Westerlund (1963); (HS): Hodge \& Sexton (1966); (C): Hodge (1975); (H88): Hodge (1988); (KMHK): Kontizas et al. (1990); (LOGLE): Pietrzynski et al. (1998, 1999); (BSDL): Bica et al. (1999); (b) Taken from Bica et al. (2008).

located outside the bar for the present study that appeared to be unstudied or were only poorly studied. Our final sample includes a total of 23 mostly unstudied clusters, eleven of which lie in the inner disc of the LMC but outside the bar. The remaining twelve are located in the outer region (Fig. 1). Here we adopt the definition presented in Bica et al. (1998) in the sense that the inner disc is that region where the mean field turnoff becomes as bright as the clump. This takes place at a deprojected radius of $\sim 4^{\circ}$. The cluster sample is presented in Table 1 , where we list the various star cluster designations from different catalogues (Col. 1), 2000.0 equatorial and galactic coordinates (Cols. 2-5), and the cluster radii given in Bica et al. (2008) (Col. 6). These radii constitute half of the mean apparent diameters obtained by computing the average between the major (a) and minor (b) axes. The last two columns of Table 1 (Cols. 7,8) list the cluster radii derived in the current study in pixels and parsecs, respectively (see Sect. 4.1).

\section{Data collection and reduction}

The observations of the selected clusters were carried out with the Víctor Blanco 4-m telescope at Cerro Tololo Inter-American Observatory (CTIO, Chile), during the nights of 2000 December 29 and 30. Washington wide-field images of about 21 LMC regions were taken with the MOSAIC II camera, which consists of an $8 \mathrm{~K} \times 8 \mathrm{~K} \mathrm{CCD}$ detector array. One pixel of the MOSAIC wide-field camera subtends $0.27^{\prime \prime}$ on the sky, resulting in a $36^{\prime} \times 36^{\prime}$ field of view. We used the Washington $C$ (Canterna 1976) and Kron-Cousins $R$ filters to be consistent with our previous studies. As shown in Geisler (1996), the $R$ filter has a significantly higher throughput than the standard Washington $T_{1}$ filter, so that $R$ magnitudes can be accurately transformed to yield $T_{1}$ magnitudes. In particular, this filter combination

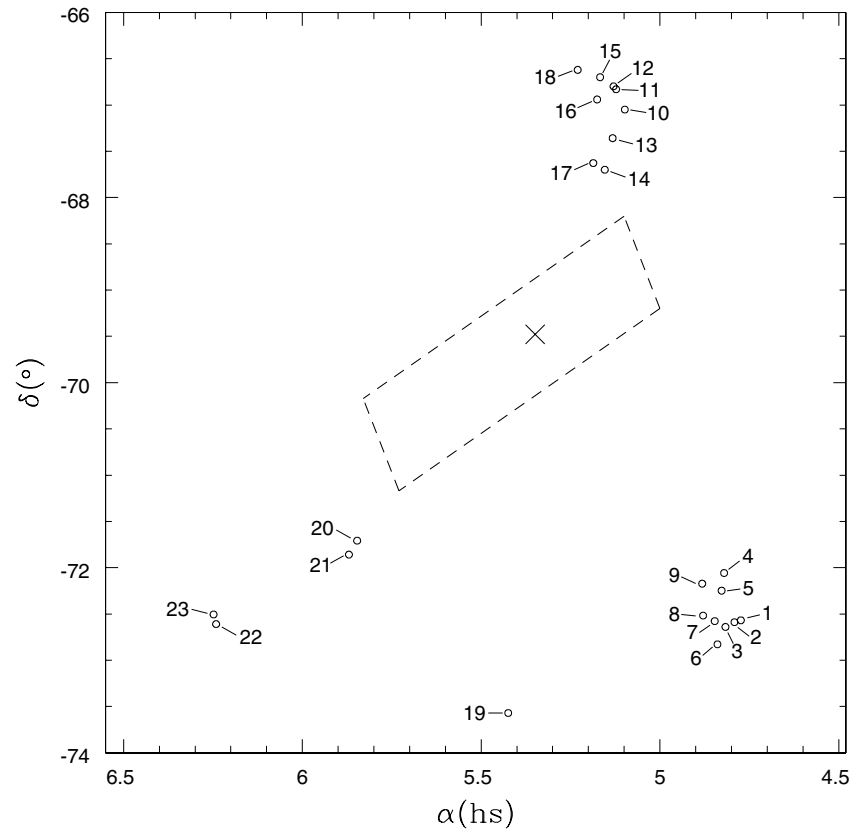

Fig. 1. Position of our target clusters (open circles) in relation to the LMC bar (dashed lines) and geometrical centre (cross). (1) SL,33, (2) SL 41, (3) KMHK 123, (4) KMHK 128, (5) LW 69, (6) KMHK 151, (7) SL 54, (8) SL 73, (9) SL 72, (10) BSDL 594, (11) BSDL 654, (12) BSDL 665, (13) BSDL 675, (14) HS 130, (15) BSDL 761, (16) BSDL 779, (17) HS 156, (18) HS 178, (19) LW 211, (20) C 11, (21) BSDL 3158, (22) KMHK 1702, (23) SL 870.

allowed us to derive accurate metallicities based on the standard giant branch method described in Geisler \& Sarajedini (1999). The Washington $C$ and Kron-Cousins $R$ filters were used with 
Table 2. Standard system mean calibration coefficients.

\begin{tabular}{lc}
\hline \hline$C$ & $T_{1}$ \\
\hline$a_{1}=(0.039 \pm 0.013)$ & $b_{1}=(-0.667 \pm 0.011)$ \\
$a_{2}=(0.249 \pm 0.007)$ & $b_{2}=(0.049 \pm 0.006)$ \\
$a_{3}=(-0.098 \pm 0.003)$ & $b_{3}=(-0.020 \pm 0.003)$ \\
\hline
\end{tabular}

typical exposure times of 450 and $150 \mathrm{~s}$, respectively. We obtained a series of bias, dome, and sky flat-field exposures per filter to calibrate the CCD instrumental signature. Standard stars of selected areas SA 98 and SA 101 from the list in Geisler (1996) were also nightly observed to standardize our photometry. SA 98 and SA 101 contain 15 and 9 standard stars, respectively, with a wide range in colour.

The MOSAIC data were reduced using the MSCRED package within IRAF $^{1}$ following the guide for mosaic reduction of Januzzi et al. (2003). Stellar photometry was performed using the stand-alone DAOPHOT II provided by Peter Stetson. For star-finding and point spread function (PSF) fitting routines, and to combine all independent measurements, we used the DAOPHOT, ALLSTAR, DAOMATCH, and DAOMASTER programs (Stetson 1987). The calibration between instrumental and standard magnitudes was obtained using the following equations:

$$
\begin{aligned}
& c=a_{1}+\left(C-T_{1}\right)+T_{1}+a_{2} X_{C}+a_{3}\left(C-T_{1}\right), \\
& r=b_{1}+T_{1}+b_{2} X_{T_{1}}+b_{3}\left(C-T_{1}\right),
\end{aligned}
$$

where $X$ is the effective airmass. Upper and lower-case letters represent standard and instrumental magnitudes, respectively. The coefficients $a_{i}$ and $b_{i}$ were derived nightly through the IRAF routine FITPARAM. The resulting mean calibration coefficients together with their errors are shown in Table 2. The nightly rms errors from the transformation to the standard system were 0.006 and $0.007 \mathrm{mag}$ for $C$ and $T_{1}$, respectively, indicating that the nights had excellent photometric quality. We finally used about 15 standard stars.

The full information gathered for each cluster consists of a running star number, the CCD $x$ and $y$ coordinates, the derived $T_{1}$ magnitude and $C-T_{1}$ colour, and the photometric errors $\sigma\left(T_{1}\right)$ and $\sigma\left(C-T_{1}\right)$. The behaviour of the $T_{1}$ and $C-T_{1}$ errors as a function of $T_{1}$ for the field of BSDL 3158 is shown in Fig. 2. Only a portion of the Washington data obtained for the star cluster SL 33 is shown here (see Table 3) for guidance regarding their form and content. The entire dataset for all clusters can be obtained as supplementary material from the on-line version of the journal.

\section{Data analysis}

\subsection{Cluster properties from star counts}

To construct density profiles of the observed clusters, we began by fitting Gaussian distributions to the star counts in the $x$ and $y$ directions to determine the coordinates of the cluster centres and their estimated uncertainties. The number of stars projected along these two directions were counted using fivepixel intervals, thus allowing us to statistically sample the spatial distributions. The fit of a single Gaussian for each projected density profile was performed using the NGAUSSFIT routine

\footnotetext{
1 IRAF is distributed by the National Optical Astronomy Observatories, which is operated by AURA, Inc., under contract with the National Science Foundation.
}

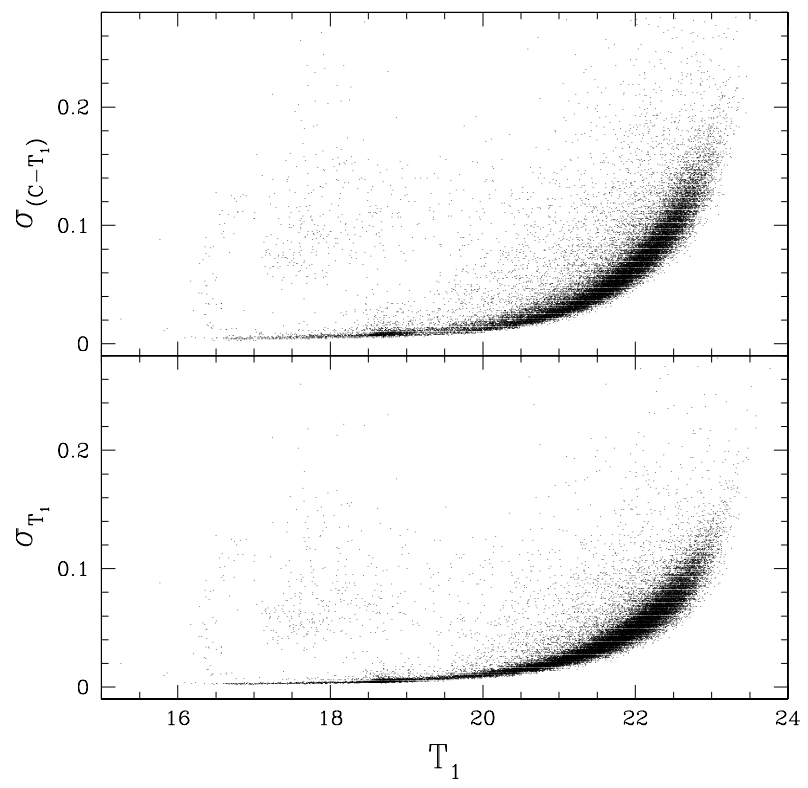

Fig. 2. $T_{1}$ magnitude and $C-T_{1}$ colour photometric errors as a function of $T_{1}$ for stars measured in the field of BSDL 3158.

Table 3. CCD $C T_{1}$ data of all stars in the field of SL 33.

\begin{tabular}{ccccccc}
\hline \hline ID & $\begin{array}{c}x \\
(\mathrm{px})\end{array}$ & $\begin{array}{c}y \\
(\mathrm{px})\end{array}$ & $\begin{array}{c}T_{1} \\
(\mathrm{mag})\end{array}$ & $\begin{array}{c}\sigma T_{1} \\
(\mathrm{mag})\end{array}$ & $\begin{array}{c}C-T_{1} \\
(\mathrm{mag})\end{array}$ & $\begin{array}{c}\sigma\left(C-T_{1}\right) \\
(\mathrm{mag})\end{array}$ \\
\hline 100 & 10.893 & 2847.002 & 22.363 & 0.060 & 0.689 & 0.071 \\
101 & 10.917 & 4041.597 & 21.874 & 0.038 & 1.165 & 0.064 \\
102 & 11.043 & 1675.679 & 20.334 & 0.012 & 1.679 & 0.022 \\
103 & 11.095 & 695.358 & 21.023 & 0.018 & 0.585 & 0.023 \\
104 & 11.106 & 3709.245 & 21.308 & 0.022 & 0.679 & 0.029 \\
105 & 11.135 & 3222.543 & 16.817 & 0.005 & 1.962 & 0.006 \\
106 & 11.155 & 1742.405 & 21.034 & 0.022 & 0.725 & 0.028 \\
107 & 11.211 & 723.802 & 21.527 & 0.025 & 0.670 & 0.035 \\
108 & 11.345 & 2543.743 & 21.005 & 0.020 & 0.526 & 0.025 \\
109 & 11.365 & 3961.926 & 21.809 & 0.035 & 0.943 & 0.050 \\
110 & 11.677 & 1627.802 & 18.183 & 0.004 & 2.153 & 0.007 \\
\hline
\end{tabular}

in the STSDAS/IRAF package. The cluster centres were determined with a typical standard deviation of \pm 5 pixels $\left(\sim 1.35^{\prime \prime}\right)$. In the particular cases in which clusters showed peculiar morphologies, like an elongation in one direction or a marked sparsity, the standard deviation turned out to be slightly higher ( \pm 10 pixels or $\left.\sim 2.7^{\prime \prime}\right)$. This is the case of clusters LW 69, SL 72, BSDL 665, and BSDL 675. Although the determination of cluster centres in these cases includes a higher degree of uncertainty, this uncertainty does not significantly change the final value obtained for the cluster radius because of the clusters' dimensions. We then built the cluster radial profiles by computing the number of stars per unit area at a given radius $r$, as shown in Fig. 3. The cluster radius $\left(r_{\mathrm{cls}}\right)$, defined as the distance from the cluster's centre where the density of stars equals that of the background, is given in pixels in Col. 7 of Table 1. Column 8 of Table 1 lists the cluster linear radii in parsecs calculated assuming that LMC is located at a distance of $50 \mathrm{kpc}$ (Subramanian \& Subramaniam 2010).

\subsection{Cluster properties from CMDs}

We show in Fig. 4 schematic images of the stars observed in the cluster fields, while in Fig. 5 we show the $\left(T_{1}, C-T_{1}\right)$ CMD of all stars measured in the field of SL 41, which is one of the most populated fields observed. The remaining cluster fields exhibit 

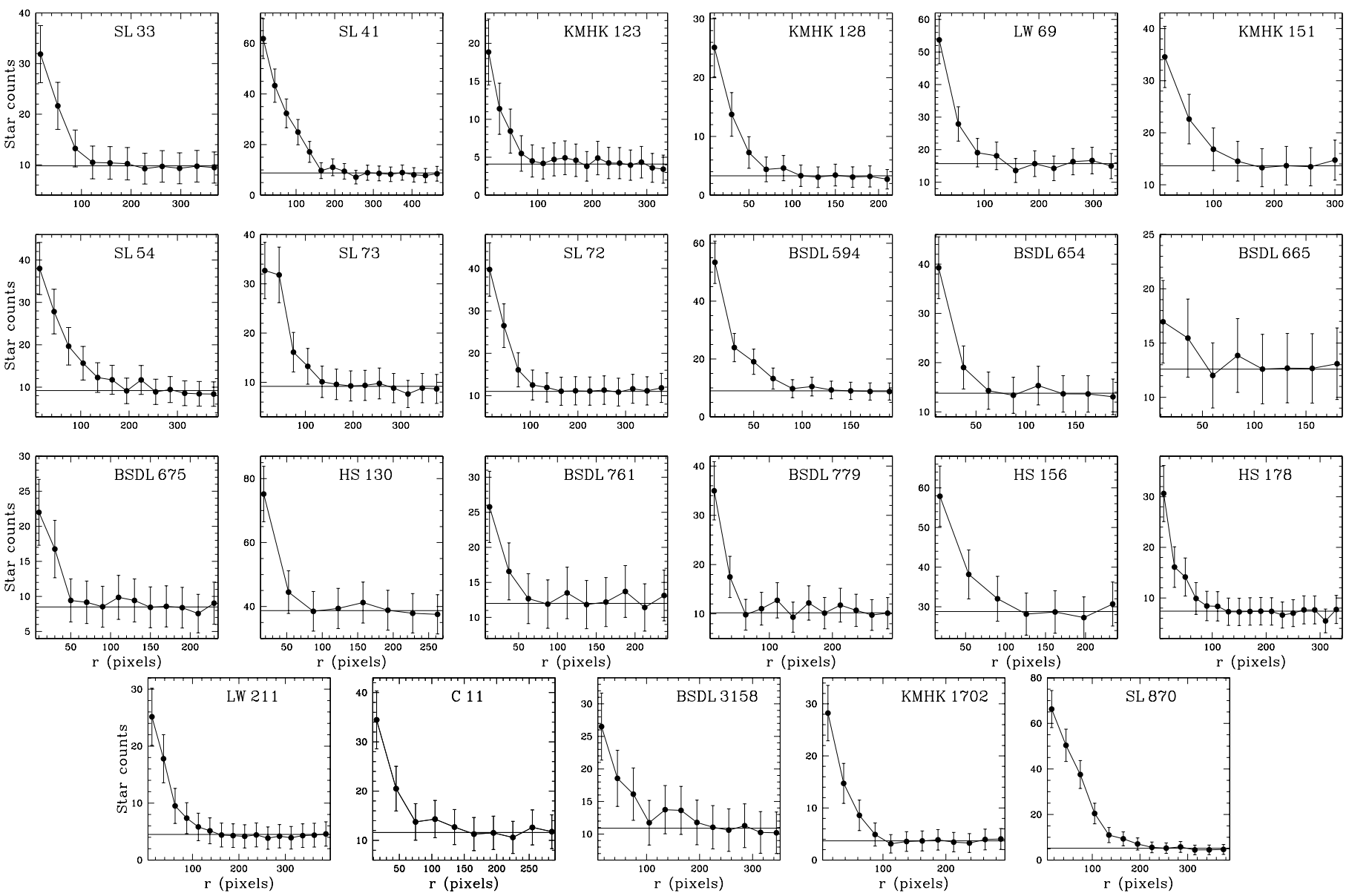

Fig. 3. Stellar density profiles for the selected clusters, with the Poisson errors included. The horizontal lines correspond to the background levels far from the clusters. The background level was determined by estimating the mean stellar density at distances larger than $\sim 300$ pixels from the centre of each cluster.

CMDs whose features vary from one cluster to another, mainly depending on age.

Since the fundamental cluster parameters estimation requires us to minimize the field-star contamination, we applied a statistical method recently developed in Piatti (priv. comm.) that is described in detail in Piatti \& Bica (2012). This procedure is briefly summarized as follows: it consists of selecting four field regions at a distance of between two and four times the cluster radius for each cluster. Then, their respective $\left(T_{1}, C-T_{1}\right)$ CMDs are obtained. The sizes of the areas of each field regions must be equal to the cluster area (generally taken as twice as large as the obtained cluster radius). Next, we count the stars lying within different intervals of magnitude-colour $\left[\delta T_{1}, \delta\left(C-T_{1}\right)\right]$ in the $\mathrm{CMD}$ of each selected region. This new method includes variable intervals, depending on how populated the studied region is. The intervals happen to be bigger in more "deserted" regions in the CMD diagrams, such as in clump regions. Conversely, they appear to be smaller in more populated regions, such as in the main sequence. Finally, the number of stars counted for each interval $\left[\delta T_{1}, \delta\left(C-T_{1}\right)\right]$ in the CMD of the surrounding field region is subtracted from the number of stars of the cluster region. For more details see Piatti \& Bica (2012). To illustrate the statistical cleaning procedure, we show in Fig. 6 the observed and cleaned CMDs for two faint star clusters of our sample BSDL 594 and SL 54.

Cluster-reddening values were estimated by interpolating the extinction maps of Burstein \& Heiles (1982). These maps were obtained from $\mathrm{HI}(21 \mathrm{~cm})$ emission data for the southern sky and provide us with foreground $E(B-V)$ colour excesses, which depend on the Galactic coordinates. As shown in Col. 3 of Table 4, the resulting $E(B-V)$ values range between 0.03 and 0.12 , values typical for the LMC. For the distance, we adopted the value of the LMC true distance modulus $(m-M)_{0}=18.50 \pm 0.10$ reported by Saha et al. (2010). On the other hand, Subramanian \& Subramaniam (2009) found that the average depth for the LMC disc is $3.44 \pm 0.16 \mathrm{kpc}$. If we consider that any cluster of the present sample could be placed in front of or behind the main body of the LMC, we conclude that the difference in apparent distance modulus could be as large as $\Delta(V-M v) \sim 0.3$ mag. Because we estimate an uncertainty of $0.2-0.3$ mag when adjusting the isochrones to the cluster CMDs, our assumption of adopting one single value for the distance modulus of all the clusters should not dominate the error budget in our final results. In fact, when we overplotted the zero-age main sequence on the observed cluster CMDs, previously shifted by the $E(B-V)$ of Table 4 and by $(m-M)_{0}=18.50$, we generally found an excellent match.

To estimate cluster ages, we used the theoretical isochrones computed by the Padova group (Girardi et al. 2002) for the Washington photometric system. These isochrones include coreovershooting effects. Although we initially used the isochrones derived by the Geneva group (Lejeune \& Schaerer 2001) which lead to nearly the same results, we finally decided to adopt Padova isochrones because they fit the fainter magnitudes of the main sequence (MS) better. We used chemical compositions of $Z=0.019,0.008$, and 0.004 , equivalent to $[\mathrm{Fe} / \mathrm{H}]=0.0,-0.4$, 


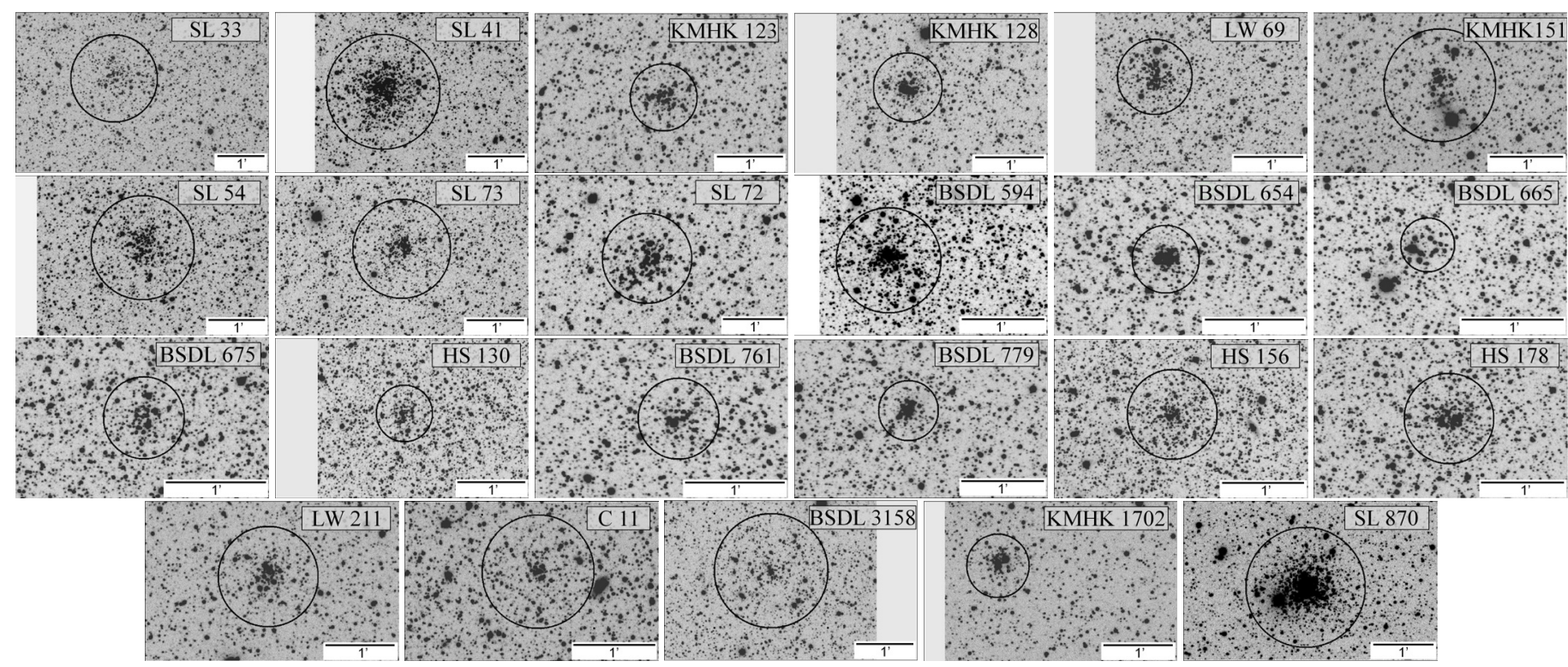

Fig. 4. Schematic images of the stars observed in the fields of our target clusters. North is up and east is to the left. The circle in each figure has been drawn with the same radius (in pixels) as the radius obtained for each cluster.

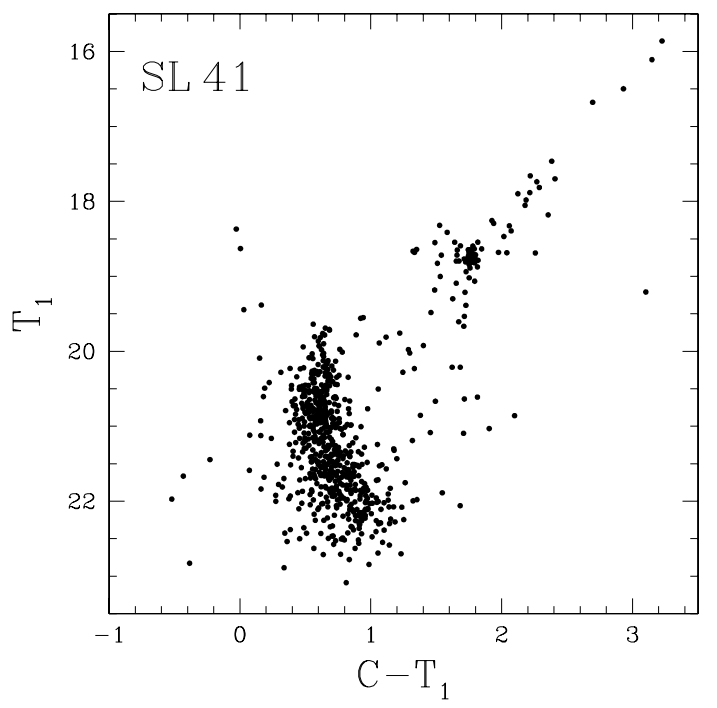

Fig. 5. Washington $\left(T_{1}, C-T_{1}\right)$ CMD for all the stars measured in the field of SL 41, one of the most populated clusters of our sample.

and -0.7 , respectively, for the isochrone sets in steps of $\Delta \log t=$ 0.05 dex. Then, we selected a set of isochrones, along with the equations $E\left(C-T_{1}\right)=1.97 E(B-V)$ and $M_{T_{1}}=T_{1}+0.58 E(B-$ $V)-\left(V-M_{V}\right)$ given in Geisler \& Sarajedini (1999), and superimposed them on the cluster $\left(T_{1}, C-T_{1}\right) \mathrm{CMDs}$, once they were properly shifted by the corresponding $E(B-V)$ colour excesses and by the LMC distance modulus. In the matching procedure, we employed different isochrones, ranging from slightly younger to slightly older than the derived cluster age. We finally adopted as the cluster age the one corresponding to the isochrone that best matched the shape and position of cluster MSs, particularly at the turn-off (TO) level. We also took into account the $T_{1}$ magnitude of the red giant clump (RGC). The age error was estimated considering the isochrones that encompassed those features best. Columns 6 and 7 of Table 4 and Fig. 7 show the results of the isochrone fittings. For each cluster CMD, we plotted the isochrone of the adopted cluster age (solid line) and two additional isochrones bracketing the derived age (dotted lines). Note
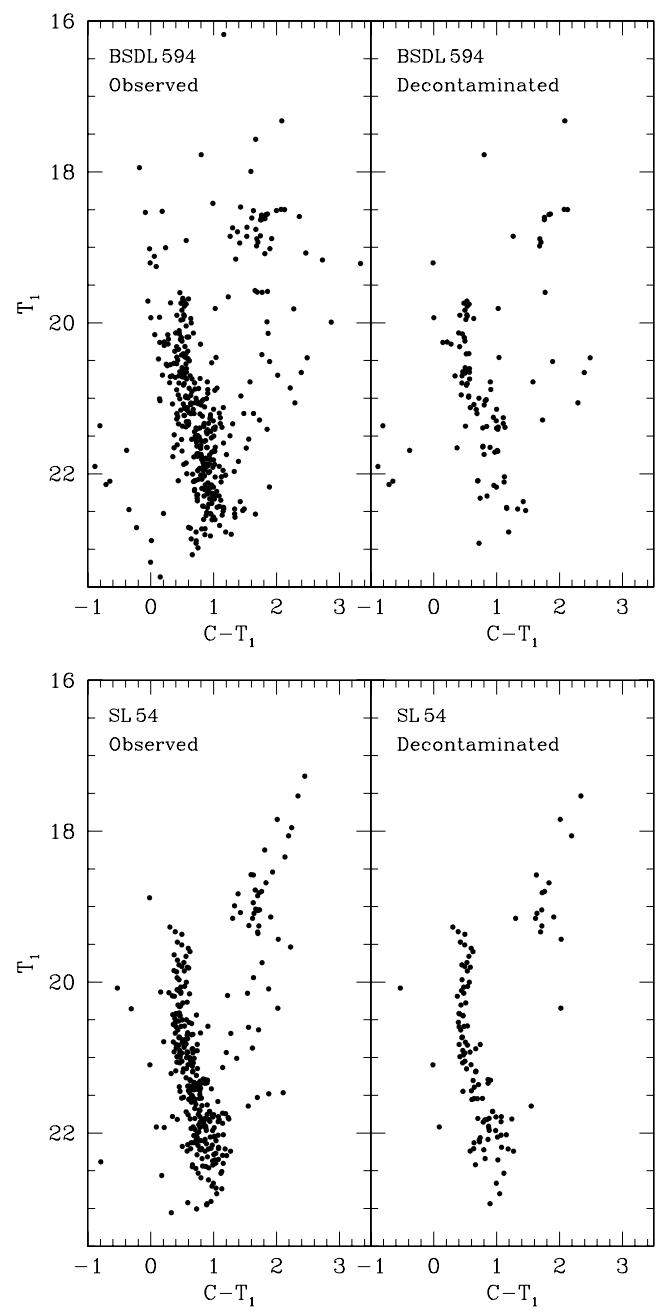

Fig. 6. Observed and cleaned CMDs of BSDL 594 and SL 54.

that the theoretically computed bluest stage during the core $\mathrm{He}$ burning phase appears to be redder than the observed RGC in the CMDs of SL 41 and SL 870, a behaviour that has also been 
A\&A 555, A131 (2013)
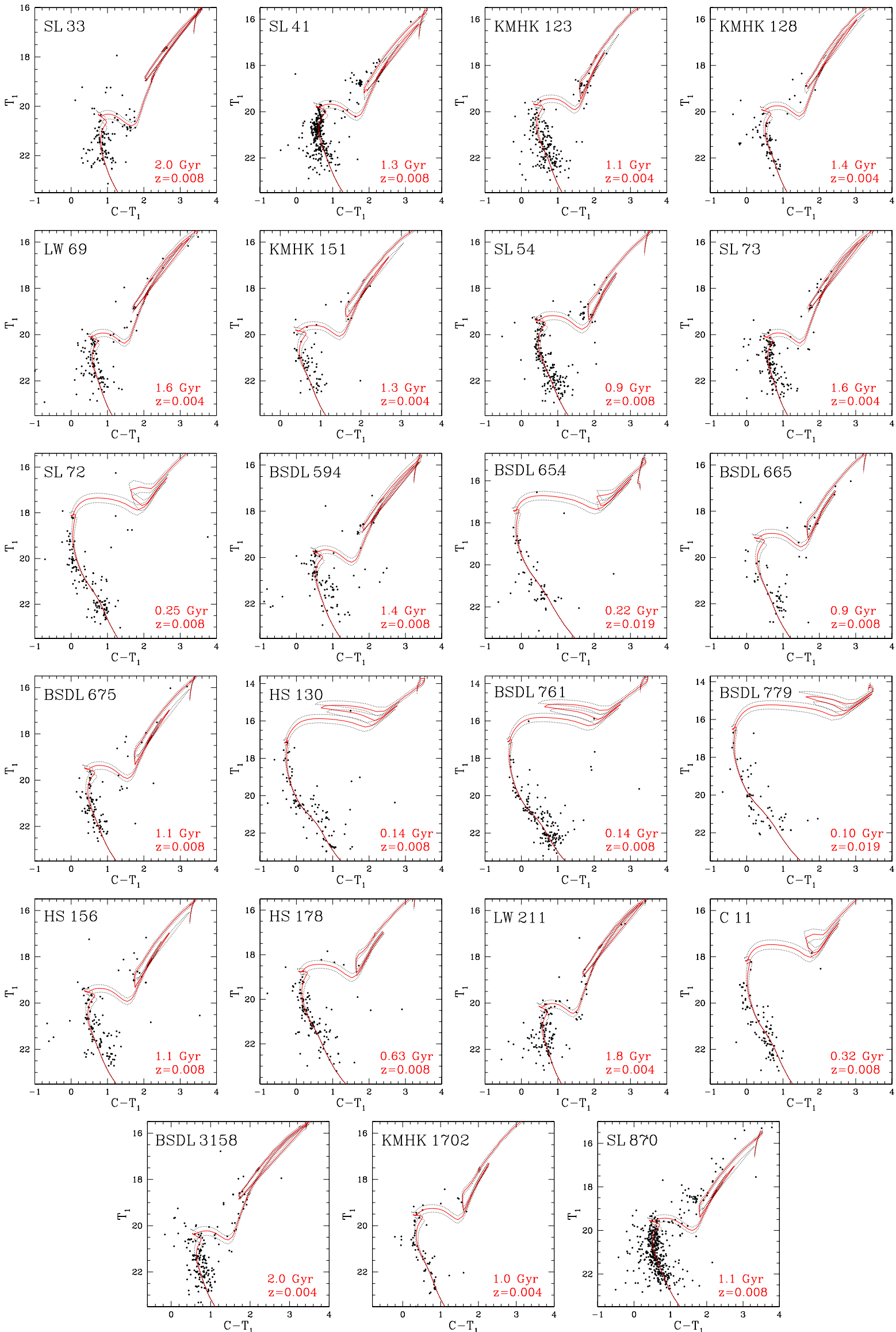

Fig. 7. Cleaned Washington $\left(T_{1}, C-T_{1}\right)$ CMDs for the studied clusters. Isochrones from Girardi et al. (2002), computed taking into account overshooting, are overplotted. The red solid and black dashed lines correspond to the derived cluster age and to the ages of the nearest younger and older isochrones. 
Table 4. Fundamental parameters of LMC clusters.

\begin{tabular}{lccccccc}
\hline \hline Name & $\begin{array}{c}\text { Deprojected } \\
\text { distance }\left(^{\circ}\right)\end{array}$ & $E(B-V)$ & $\delta T_{1}$ & $\begin{array}{c}\delta T_{1} \text { age } \\
(\mathrm{Gyr})\end{array}$ & $\begin{array}{c}\text { Isochrone age } \\
(\mathrm{Gyr})\end{array}$ & {$[\mathrm{Fe} / \mathrm{H}]_{\text {isochrone }}$} & {$[\mathrm{Fe} / \mathrm{H}]_{\mathrm{SGB}}$} \\
\hline SL 33 & 5.1 & 0.12 & - & - & $2.0 \pm 0.2$ & -0.4 & -0.6 \\
SL 41 & 5.1 & 0.12 & 1.0 & $1.4 \pm 0.4$ & $1.3 \pm 0.1$ & -0.4 & - \\
KMHK 123 & 5.0 & 0.12 & - & - & $1.1 \pm 0.1$ & -0.7 & - \\
KMHK 128 & 5.4 & 0.11 & - & - & $1.4 \pm 0.2$ & -0.7 & - \\
LW 69 & 4.6 & 0.12 & 1.4 & $1.7 \pm 0.4$ & $1.6 \pm 0.2$ & -0.7 & - \\
KMHK 151 & 5.1 & 0.12 & 1.1 & $1.4 \pm 0.4$ & $1.3 \pm 0.1$ & -0.7 & - \\
SL 54 & 4.9 & 0.12 & - & - & $0.9 \pm 0.1$ & -0.4 & - \\
SL 73 & 4.7 & 0.12 & 1.3 & $1.6 \pm 0.4$ & $1.6 \pm 0.2$ & -0.7 & - \\
SL 72 & 4.4 & 0.13 & - & - & $0.25 \pm 0.03$ & -0.4 & - \\
BSDL 594 & 3.4 & 0.05 & 0.8 & $1.3 \pm 0.4$ & $1.4 \pm 0.2$ & -0.4 & - \\
BSDL 654 & 3.6 & 0.03 & - & - & $0.22 \pm 0.03$ & 0.0 & - \\
BSDL 665 & 3.6 & 0.03 & - & - & $0.9 \pm 0.1$ & -0.4 & - \\
BSDL 675 & 3.0 & 0.06 & 1.0 & $1.4 \pm 0.4$ & $1.1 \pm 0.1$ & -0.4 & - \\
HS 130 & 2.5 & 0.06 & - & - & $0.14 \pm 0.02$ & -0.4 & - \\
BSDL 761 & 3.6 & 0.04 & - & - & $0.14 \pm 0.02$ & -0.4 & - \\
BSDL 779 & 3.3 & 0.04 & - & - & $0.10 \pm 0.01$ & 0.0 & - \\
HS 156 & 2.5 & 0.06 & 0.9 & $1.3 \pm 0.4$ & $1.1 \pm 0.1$ & -0.4 & - \\
HS 178 & 3.5 & 0.04 & - & - & $0.63 \pm 0.07$ & -0.4 & - \\
LW 211 & 4.7 & 0.10 & - & - & $1.8 \pm 0.2$ & -0.7 & - \\
C11 & 3.4 & 0.10 & - & - & $0.32 \pm 0.05$ & -0.4 & - \\
BSDL 3158 & 3.5 & 0.10 & 1.7 & $2.1 \pm 0.4$ & $2.0 \pm 0.2$ & -0.7 & -0.8 \\
KMHK 1702 & 5.3 & 0.11 & 0.7 & $1.2 \pm 0.4$ & $1.0 \pm 0.1$ & -0.7 & - \\
SL 870 & 5.4 & 0.09 & 0.7 & $1.2 \pm 0.4$ & $1.1 \pm 0.1$ & -0.4 & - \\
\hline
\end{tabular}

detected in previous studies (see, e.g., Geisler et al. 2003; Piatti et al. 2004; Clariá et al. 2007).

A second method to derive cluster ages is based on the $\delta\left(T_{1}\right)$ parameter, defined as the difference in $T_{1}$ magnitude between the RGC and the MSTO in the Washington $\left(T_{1}, C-T_{1}\right)$ CMD. The age is obtained from the following equation given in Geisler et al. (1997):

$\operatorname{Age}(\mathrm{Gyr})=0.23+2.31 \times \delta T_{1}-1.80 \times \delta T_{1}^{2}+0.645 \times \delta T_{1}^{3}$,

with a typical error of $\pm 0.3 \mathrm{Gyr}$. Age determination via $\delta T_{1}$, however, is applicable only to intermediate-age (IACs) and/or old clusters, i.e. generally older than 1 Gyr. Although some clusters seemed to be IACs (1-3 Gyr), it was not possible to determine their ages from $\delta T_{1}$ because the RGC in their CMDs was not clearly detected. This is because sometimes the central regions of the clusters appear to be saturated, perhaps there are just very few RC stars in some faint clusters, or else they are not photometrically well resolved in our images. In these cases, the red giant stars are missed and no clump is visible in the CMDs. This is why we derived ages of only ten clusters older than $1 \mathrm{Gyr}$ based on the $\delta T_{1}$ parameter. The mean $\delta T_{1}$ values were estimated from the average of independent measurements made by two authors. These measurements agreed in general very well. The resulting $\delta T_{1}$ values and the corresponding cluster ages are listed in Cols. 4 and 5 of Table 4 . As can be seen, $\delta T_{1}$ ages agree well with those estimated from the isochrone fitting, which confirms that both procedures allow us to estimate ages on a similar scale.

Pandey et al. (2010) studied the integrated magnitudes and colours for some LMC clusters from synthetic models, among which are BSDL 654, BSDL 675, and BSDL 779. They report ages of 0.16 and 1 Gyr for BSDL 654 and BSDL 675, respectively. These age estimates agree well with ours within the errors (see Table 4). For BSDL 779, however, these authors reported an age of $0.03 \mathrm{Gyr}$, i.e., substantially younger than the one obtained here. On the other hand, two clusters from our sample, SL 870 and SL 41, were classified in Bica et al. (1996) as belonging to SWB V type (Searle et al. 1980), based on their integrated $(B-V)$ and $(U-B)$ colours. This SWB V type is compatible with clusters belonging to the $0.8-2.0 \mathrm{Gyr}$ range, which agrees well with the age values derived here for these two clusters.

Metallicities for only the two oldest clusters of our sample (SL 33 and BSDL 3158) were also obtained using the $\left[M_{T_{1}},(C-\right.$ $\left.T_{1}\right)_{0}$ ] plane with the standard giant branches (SGBs) of Geisler \& Sarajedini (1999). As these authors claim, this method should be applied only to star clusters aged 2 Gyr or older. Geisler and Sarajedini demonstrated that the metallicity sensitivity of the SGBs (each giant branch corresponds to an isoabundance curve) is three times higher than that of the $V, I$ technique (Da Costa \& Armandroff 1990) and that, consequently, it is possible to determine metallicities three times more precisely for a given photometric error. We followed the SGB procedure of inserting absolute $M_{T_{1}}$ magnitudes and intrinsic $\left(C-T_{1}\right)_{0}$ colours for the clusters into Fig. 4 of Geisler \& Sarajedini (1999) to roughly derive their metal abundances $([\mathrm{Fe} / \mathrm{H}])$ by interpolation (Fig. 8$)$. The derived metallicities were corrected for age effects following the prescriptions given in Geisler et al. (2003). The final agecorrected metallicities for SL 33 and BSDL 3158 are listed in Col. 8 of Table 4 . They agree very well with the $Z$ values associated with the isochrones that best resemble the cluster features. The Girardi et al. (2002) models are computed for $[\mathrm{Fe} / \mathrm{H}]=-1.3$ and -0.7 dex, but not for intermediate-metallicity values. We finally adopted for our cluster sample averaged values of the ages and metallicities derived from the two different procedures.

\section{Discussion}

The present sample includes 23 mostly unstudied LMC clusters within the narrow age range of $0.1-2.1 \mathrm{Gyr}$, with metallicities between 0.0 and -0.8 dex. To search for possible variations of ages and metallicities as a function of cluster position in the LMC, we calculated deprojected angular distances for our sample using Eq. (1) of Clariá et al. (2005), assuming that all clusters are part of the LMC inclined disc. We adopted $i \approx 35.8^{\circ}$ and $p=145^{\circ}$ for the tilt of the LMC plane and the position angle of 

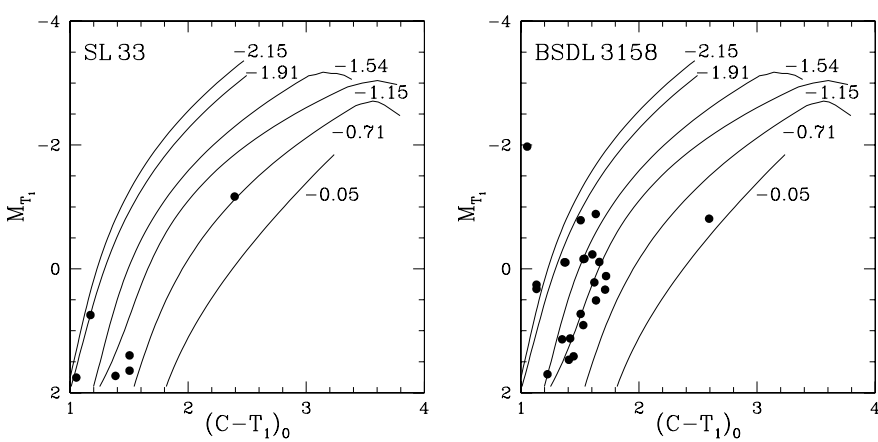

Fig. 8. Washington $M_{T_{1}}$ vs. $\left(C-T_{1}\right)_{0}$ diagram of upper RGB stars in two LMC star clusters, with SGBs taken from Geisler \& Sarajedini (1999) superimposed. An age-dependent correction to the indicated metallicities was applied for these clusters.

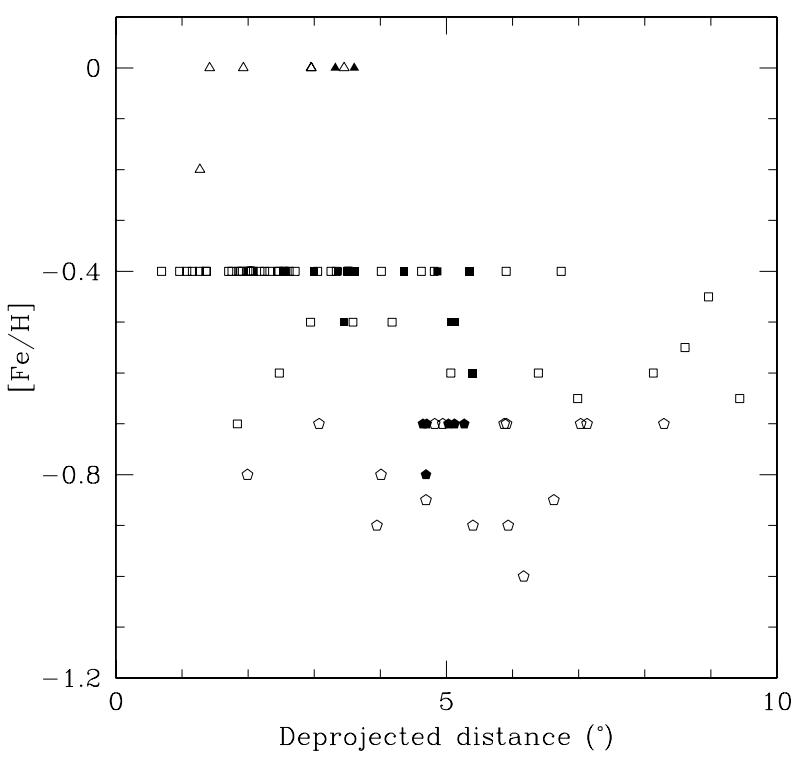

Fig. 9. Metallicities as a function of deprojected angular distances from the LMC centre for the 23 studied clusters (filled symbols). Clusters included in Table 5 are represented by open symbols. Triangles, squares, and pentagons correspond to the following $[\mathrm{Fe} / \mathrm{H}]$ intervals: $[\mathrm{Fe} / \mathrm{H}]>$ $-0.4 ;-0.4 \geq[\mathrm{Fe} / \mathrm{H}]>-0.7$, and $[\mathrm{Fe} / \mathrm{H}] \leq-0.7$, respectively. The old cluster ESO 121-03 has not been plotted.

the line of nodes, respectively (Olsen \& Salyk 2002). The resulting deprojected angular distances are listed in Col. 2 of Table 4.

Figure 9 shows that in the comparatively small range of deprojected angular distances considered here $\left(2.5^{\circ}-6^{\circ}\right)$, the most metal-rich clusters of our sample (filled symbols) tend to lie closer to NGC 1928, whose position was adopted as the LMC centre $\left(\alpha_{2000}=5^{\mathrm{h}} 20^{\mathrm{m}} 57^{\mathrm{s}}, \delta_{2000}=-69^{\circ} 28^{\prime} 41^{\prime \prime}\right)$. Filled symbols of Fig. 10 show how our derived cluster ages vary as a function of the deprojected distances. As the clusters become older, their corresponding deprojected distances tend to be proportionally larger, thus supporting previous results in similar ranges of deprojected angular distances for clusters (e.g., Piatti et al. 2009) and field stars (Piatti \& Geisler 2013).

To examine what the above mentioned position-agemetallicity relationships are like when the range of deprojected angular distances is enlarged, we searched in the literature for previous LMC cluster studies in which ages and metallicities were determined on a similar scale as that of the present cluster sample. We found a total of 76 LMC star clusters with ages and metallicities derived from the Washington $C, T_{1}$ technique

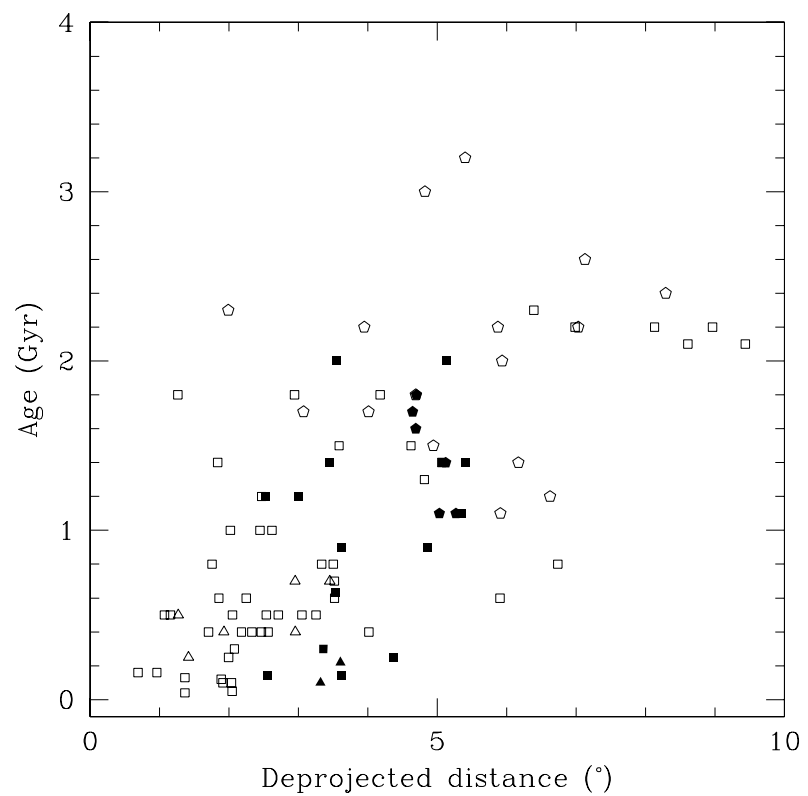

Fig. 10. Ages as a function of deprojected angular distances from the LMC centre for the 23 studied clusters (filled symbols). Clusters included in Table 5 are represented by open symbols. Triangles, squares, and pentagons correspond to the following $[\mathrm{Fe} / \mathrm{H}]$ intervals: $[[\mathrm{Fe} / \mathrm{H}]>$ $-0.4 ;-0.4 \geq[\mathrm{Fe} / \mathrm{H}]>-0.7$, and $[\mathrm{Fe} / \mathrm{H}] \leq-0.7$, respectively. The old cluster ESO 121-03 has not been plotted.

using CTIO telescopes and applying the same methods as in the current study (Table 5). Our 23 clusters increase the current total amount of LMC clusters using Washington photometry by $\sim 30 \%$. We calculated deprojected distances for these additional 76 clusters and included them in Col. 2 of Table 5. We plot in Figs. 9 and 10 their ages and metallicities as a function of their deprojected distances for three metallicity intervals, $[\mathrm{Fe} / \mathrm{H}]>-0.4$ (triangles), $-0.4 \geq[\mathrm{Fe} / \mathrm{H}]>-0.7$ (squares), and $[\mathrm{Fe} / \mathrm{H}] \leq-0.7$. When the new 76 clusters are added, the range of deprojected distances increases by a factor of $\sim 2.5$. Figure 9 now reveals that although the most metal-rich clusters tend to be located closer to the galaxy centre, clusters with $[\mathrm{Fe} / \mathrm{H}]<-0.4$ appear to be distributed over the entire LMC disc, with a high dispersion. This fact reinforces the idea of the nonexistence of a radial metallicity gradient in the LMC, as suggested in several previous studies (e.g., Grocholski et al. 2006; Carrera et al. 2008; Piatti et al. 2009). Figure 10 includes clusters younger than 3.2 Gyr, since ESO 121-03 ( 8.5 Gyr) has not been plotted. As expected, the most metal-poor clusters turn out to be also the older ones. Moreover, cluster formation seems to be concentrated in the inner LMC disc, since younger clusters were formed closer to the LMC centre than the older ones.

\section{Summary and conclusions}

We continued the systematic study of star clusters of the LMC carried out using the Washington photometric system. We presented $\left(T_{1}, C-T_{1}\right)$ CMDs for 23 star clusters that lie within the inner disc and outer regions of the LMC. Only five of these clusters have previous age estimates in the literature. However, no metallicity estimate whatsoever has been found for any cluster of our sample. Ages and metallicities were determined by two different methods. We compared the CMDs with theoretical isochrones in the Washington system and also estimated ages using the magnitude difference between the RGC and 
Table 5. Ages and metallicities of LMC star clusters derived from Washington photometry.

\begin{tabular}{|c|c|c|c|c|}
\hline Cluster name & $\begin{array}{l}\text { Deprojected } \\
\text { distance }\left({ }^{\circ}\right)\end{array}$ & Age (Gyr) & {$[\mathrm{Fe} / \mathrm{H}]$} & Reference \\
\hline SL 8 & 4.2 & $1.6 / 1.8$ & -0.5 & 1,2 \\
\hline NGC 1697 & 3.5 & $0.7 \pm 0.1$ & 0.0 & 3 \\
\hline HS 38 & 4.0 & $0.4 \pm 0.1$ & -0.4 & 4 \\
\hline КМНК 229 & 2.6 & $1.0 \pm 0.2$ & -0.4 & 4 \\
\hline H88-26 & 3.3 & $0.8 \pm 0.2$ & -0.4 & 4 \\
\hline H88-40 & 3.5 & $0.7 \pm 0.2$ & -0.4 & 4 \\
\hline SL 126 & 8.9 & $1.9 / 2.2$ & -0.45 & 1,2 \\
\hline SL 133 & 5.9 & $2.2 \pm 0.3$ & -0.7 & 3 \\
\hline H88-55 & 3.3 & $0.5 \pm 0.1$ & -0.4 & 4 \\
\hline SL 154 & 3.0 & $0.5 \pm 0.1$ & -0.4 & 4 \\
\hline КМHK 506 & 2.2 & $0.6 \pm 0.1$ & -0.4 & 4 \\
\hline SL 218 & 2.0 & $0.05 \pm 0.01$ & -0.4 & 5 \\
\hline NGC 1836 & 1.9 & $0.4 \pm 0.1$ & 0.0 & 6 \\
\hline BRHT 4b & 1.9 & $0.10 \pm 0.02$ & -0.4 & 5 \\
\hline NGC 1839 & 1.9 & $0.12 \pm 0.02$ & -0.4 & 5 \\
\hline NGC 1838 & 2.0 & $0.10 \pm 0.02$ & -0.4 & 5 \\
\hline SL 229 & 2.1 & $0.3 \pm 0.1$ & -0.4 & 4 \\
\hline SL 244 & 1.8 & $1.6 / 1.4 \pm 0.3$ & -0.7 & 1,8 \\
\hline BSDL 716 & 2.2 & $0.4 \pm 0.1$ & -0.4 & 4 \\
\hline SL 262 & 8.6 & 2.1 & -0.55 & 1,2 \\
\hline HS 151 & 1.8 & $0.8 \pm 0.2$ & -0.4 & 4 \\
\hline NGC 1860 & 1.4 & $0.25 \pm 0.5$ & 0.0 & 6 \\
\hline H88-188 & 2.7 & $0.5 \pm 0.1$ & -0.4 & 4 \\
\hline HS 154 & 2.5 & $0.5 \pm 0.1$ & -0.4 & 4 \\
\hline SL 293 & 2.5 & $0.4 \pm 0.1$ & -0.4 & 4 \\
\hline NGC 1863 & 1.4 & $0.04 \pm 0.01$ & -0.4 & 5 \\
\hline SL 300 & 2.6 & $0.4 \pm 0.1$ & -0.4 & 4 \\
\hline NGC 1865 & 1.3 & $0.9 / 0.5 \pm 0.1$ & -0.2 & 1,6 \\
\hline BSDL 1024 & 1.0 & $0.16 \pm 0.03$ & -0.4 & 4 \\
\hline BSDL 1035 & 1.2 & $0.5 \pm 0.1$ & -0.4 & 4 \\
\hline H88-245 & 0.7 & $0.16 \pm 0.04$ & -0.4 & 4 \\
\hline SL 351 & 1.1 & $0.5 \pm 0.1$ & -0.4 & 4 \\
\hline SL 359 & 1.3 & $1.8 \pm 0.3$ & -0.4 & 1,8 \\
\hline SL 388 & 7.0 & $2.6 / 2.2$ & -0.65 & 1,2 \\
\hline IC 2134 & 6.9 & 1.0 & - & 2 \\
\hline SL 451 & 7.0 & 2.2 & -0.7 & 1,2 \\
\hline SL 446A & 2.0 & $2.3 \pm 0.3$ & -0.8 & 1,8 \\
\hline SL 444 & 2.0 & $0.5 \pm 0.1$ & -0.4 & 6 \\
\hline SL 490 & 4.8 & $1.3 \pm 0.3$ & -0.4 & 7 \\
\hline SL 505 & 2.5 & $1.6 / 1.2 \pm 0.3$ & -0.6 & 1,8 \\
\hline SL 510 & 1.4 & $0.13 \pm 0.03$ & -0.4 & 4 \\
\hline SL 509 & 6.6 & $1.4 / 1.2$ & -0.85 & 1,2 \\
\hline LW 224 & 3.0 & $0.7 \pm 0.1$ & 0.0 & 6 \\
\hline LW 231 & 6.7 & $0.8 \pm 0.3$ & -0.4 & 7 \\
\hline NGC 1997 & 7.1 & $2.6 \pm 0.5$ & -0.7 & 3 \\
\hline SL 548 & 3.0 & $0.4 \pm 0.1$ & 0.0 & 6 \\
\hline SL 555 & 3.1 & $1.6 / 1.7 \pm 0.3$ & -0.7 & 1,8 \\
\hline SL 549 & 5.9 & $1.3 / 2.0 \pm 0.3$ & -0.9 & 1,8 \\
\hline КМHK 1045 & 1.9 & $0.6 \pm 0.1$ & -0.4 & 4 \\
\hline КМHK 1055 & 2.0 & $1.0 \pm 0.2$ & -0.4 & 4 \\
\hline SL 588 & 1.7 & $0.4 \pm 0.1$ & -0.4 & 4 \\
\hline NGC 2093 & 2.0 & $0.25 \pm 0.05$ & -0.4 & 4 \\
\hline SL 663 & 4.8 & $3.0 \pm 0.8$ & -0.7 & 3 \\
\hline SL 674 & 3.9 & $2.1 / 2.2 \pm 0.3$ & -0.9 & 1,8 \\
\hline SL 678 & 4.0 & $1.8 \pm 0.3$ & -0.8 & 8 \\
\hline H88-333 & 2.3 & $0.4 \pm 0.1$ & -0.4 & 4 \\
\hline BSDL 2995 & 2.4 & $1.0 \pm 0.2$ & -0.4 & 4 \\
\hline $\mathrm{H} 7$ & 3.2 & 1.4 & - & 1 \\
\hline SL 769 & 2.9 & 1.8 & -0.5 & 2 \\
\hline КМHK 1507 & 3.5 & $0.8 \pm 0.3$ & -0.4 & 7 \\
\hline SL 775 & 3.5 & $0.6 \pm 0.3$ & -0.4 & 7 \\
\hline OHSC 28 & 8.3 & $2.4 \pm 0.5$ & -0.7 & 3 \\
\hline NGC 2161 & 5.9 & $1.1 \pm 0.3$ & -0.7 & 9 \\
\hline NGC 2153 & 4.6 & 1.3 & - & 1 \\
\hline
\end{tabular}

Table 5. continued.

\begin{tabular}{lcccc}
\hline \hline Cluster name & $\begin{array}{c}\text { Deprojected } \\
\text { distance }\left({ }^{\circ}\right)\end{array}$ & Age $(\mathrm{Gyr})$ & {$[\mathrm{Fe} / \mathrm{H}]$} & Reference \\
\hline NGC 2155 & 5.4 & $3.2 \pm 0.6$ & -0.9 & 10 \\
SL 817 & 3.6 & $2.5 / 1.5$ & -0.5 & 1,2 \\
ESO 121-03 & 10.4 & 8.5 & -1.05 & 1,2 \\
SL 842 & 8.1 & $1.9 / 2.2$ & -0.6 & 1,2 \\
NGC 2213 & 4.6 & 1.5 & -0.4 & 11 \\
SL 862 & 4.7 & 1.8 & -0.85 & 1,2 \\
OHSC 33 & 6.2 & $1.2 / 1.4$ & -1.0 & 1,2 \\
SL 874 & 4.9 & $1.5 \pm 0.3$ & -0.7 & 9 \\
KMHK 1719 & 5.1 & $1.4 \pm 0.3$ & -0.6 & 9 \\
LW 469 & 5.9 & $0.6 \pm 0.1$ & -0.4 & 4 \\
SL 896 & 6.4 & $2.3 \pm 0.3$ & -0.6 & 10 \\
OHSC 37 & 9.4 & 2.1 & -0.65 & 1,2 \\
\hline
\end{tabular}

References. (1) Geisler et al. (1997); (2) Bica et al. (1998); (3) Piatti et al. (2009); (4) Piatti (2012); (5) Piatti et al. (2003b); (6) Piatti et al. (2003a); (7) Palma et al. (2011); (8) Geisler et al. (2003); (9) Piatti et al. (2011); (10) Piatti et al. (2002); (11) Geisler (1987).

the MSTO, and derived metallicities by comparing the giant branches with standard calibrating clusters. The two methods applied for determining ages and metallicities agree well with each other. Fourteen clusters are found to be IACs (1-3 Gyr), with $[\mathrm{Fe} / \mathrm{H}]$ values ranging from -0.4 to -0.7 . The remaining nine objects are younger than $1 \mathrm{Gyr}$, with metallicities between 0.0 and -0.4 . By combining the current results with those of a sample of 76 additional clusters with ages and metallicities derived on a scale similar to that of the present work, we confirmed previous findings regarding the chemical evolution of the LMC. We found no evidence of a metallicity gradient and also found that the younger clusters were formed closer to the LMC centre than the older ones.

Acknowledgements. We thank the staff and personnel at CTIO for hospitality and assistance during the observations. We gratefully acknowledge financial support from the Argentinian institutions CONICET, FONCYT and SECYT (Universidad Nacional de Córdoba). D.G. gratefully acknowledges support from the Chilean BASAL Centro de Excelencia en Astrofísica y Tecnologías Afines (CATA) grant PFB-06/2007. This work is based on observations made at Cerro Tololo Inter-American Observatory, which is operated by AURA, Inc., under cooperative agreement with the National Science Foundation. T.P. wishes to thank L. Bassino, R. Leiton and L. Macri for their valuable guidance and discussion in the reduction process of MOSAIC data. We appreciate the valuable comments and suggestions of the anonimous referee, which helped us to improve the manuscript. This research has made use of the SIMBAD database, operated at CDS, Strasbourg, France; also the SAO/NASA Astrophysics data (ADS).

\section{References}

Baumgardt, H., Parmentier, G., Anders, P., \& Grebel, E. K. 2013, MNRAS, 430, 676

Bica, E., Clariá, J. J., Dottori, H., Santos, J. F. C., Jr. \& Piatti, A. E. 1996, ApJS, 102,57

Bica, E., Geisler, D., Dottori, H., et al. 1998, AJ, 116, 723

Bica, E., Schmitt, H. R., Dutra, C. M., \& Oliveira, H. L. 1999, AJ, 117, 238

Bica, E., Bonatto, C., Dutra, C. M., \& Santos, J. F. C., Jr. 2008, MNRAS, 389, 678

Burstein, D., \& Heiles, C. 1982, AJ, 87, 1165

Canterna, R. 1976, AJ, 81, 228

Carrera, R., Gallart, C., Hardy, E., Aparicio, A., \& Zinn, R. 2008, AJ, 135, 836 Clariá, J. J., Piatti, A. E., Santos, J. F. C., Jr., et al. 2005, BAAA, 48, 140

Clariá, J. J., Piatti, A. E., Parisi, M. C., \& Ahumada, A. V. 2007, MNRAS, 379, 159

Da Costa, G. S., \& Armandroff, T. E. 1990, AJ, 100, 162

Geisler, D. 1987, AJ, 93, 1081

Geisler, D. 1996, AJ, 111, 480

Geisler, D., \& Sarajedini, A. 1999, AJ, 117, 308

Geisler, D., Bica, E., Dottori, H., et al. 1997, AJ, 114, 1920 
A\&A 555, A131 (2013)

Geisler, D., Piatti, A. E., Bica, E. \& Clariá, J. J. 2003, MNRAS, 341, 771

Girardi, L., Bertelli, G., Bressan, A., et al. 2002, A\&A, 391, 195

Grocholski, A. J., Cole, A. A., Sarajedini, A., Geisler, D., \& Smith, V. V. 2006, AJ, 132, 1630

Hodge, P. W. 1975, Ir. Astron. J., 12, 77

Hodge, P. W. 1988, PASP, 100, 1051

Hodge, P. W., \& Sexton, J. A. 1966, AJ, 71, 363

Januzzi, B. T., Claver, J., \& Valdes, F. 2003, The NOAO Deep Wide-Field Survey MOSAIC Data Reductions

http: //www . noao.edu/noao/noaodeep/Reduction0pt/frames.html Kontizas, M., Morgan, D. H., Hatzidimitriou, D., \& Kontizas, E. 1990, A\&AS, 84,527

Lejeune, T., \& Schaerer, D. 2001, A\&A, 366, 538

Lynga, G., \& Westerlund, B. E. 1963, MNRAS, 127, 31

Olsen, K. A. G., \& Salyk, C. 2002, AJ, 124, 2045

Palma, T., Clariá, J. J., Geisler, D., et al. 2011, BAAA, 54, 195

Pandey, A. K., Sandhu, T. S., Sagar, R., \& Battinelli, P. 2010, MNRAS, 403, 1491
Piatti, A. E. 2012, A\&A, 540, A58

Piatti, A. E., \& Bica, E. 2012, MNRAS, 425, 3085

Piatti, A. E., \& Geisler, D. 2013, AJ, 145, 17

Piatti, A. E., Sarajedini, A., Geisler, D., Bica, E., \& Clariá, J. J. 2002, MNRAS, 329,556

Piatti, A. E., Bica, E., Geisler, D., \& Clariá, J. J. 2003a, MNRAS, 344, 965 Piatti, A. E., Geisler, D., Bica, E., \& Clariá, J. J. 2003b, MNRAS, 343, 851 Piatti, A. E., Clariá, J. J., \& Ahumada, A. V. 2004, A\&A, 421, 991

Piatti, A. E., Geisler, D., Sarajedini, A., \& Gallart, C. 2009, A\&A, 501, 585

Piatti, A. E., Clariá, J. J., Parisi, M. C., \& Ahumada, A. V. 2011, PASP, 123, 519

Pietrzynski, G., Udalski, A., Kubiak, M., et al. 1998, Acta Astron., 48, 175

Pietrzynski, G., Udalski, A., Kubiak, M., et al. 1999, Acta Astron., 49, 521

Saha, A., Olszewski, E. W., Brondel, B., et al. 2010, AJ, 140, 1719

Searle, L., Wilkinson, A., \& Bagnuolo, W. G. 1980, ApJ, 239, 803

Shapley, H., \& Lindsay, E. M. 1963, Ir. Astron. J., 6, 74

Stetson, P. B. 1987, PASP, 99, 191

Subramanian, S., \& Subramaniam, A. 2009, A\&A, 496, 399

Subramanian, S., \& Subramaniam, A. 2010, A\&A, 520, A24 